\title{
A Fault Detection and Faulted-Phase Selection Approach for Transmission Lines with Haar Wavelet Transform
}

\author{
Joe-Air Jiang, Member, IEEE, Ping-Lin Fan, Ching-Shan Chen, Chi-Shan Yu, and Jin-Yi Sheu
}

\begin{abstract}
Wavelet transform is a novel signal processing technique and has been widely used in many applications, including power system disturbance analysis. Many published woks introduce the wavelet transform as a tool to analyze power system disturbances. In this work, a dyadic wavelet transform based approach, which is used to detect transmission line faults, is proposed. The coefficient of discrete approximation of the dyadic wavelet transform with Haar wavelet is used to be an index for transmission line fault detection and faulted-phase selection. Basic ideas and the proposed algorithm are described in this paper. MATLAB/Simulink is used to generate fault signals and verify the correctness of the algorithm. Simulation results reveal that the performance of the proposed fault detection indicator is promising and easy to implement for computer relaying application.
\end{abstract}

Index Terms- Fault detection, faulted-phase selection, Haar wavelet, wavelet transform.

\section{INTRODUCTION}

$\mathrm{W}$ avelet transform (WT) is a novel signal processing technique developed from the Fourier transform (FT) and has been widely used to signal processing application [1]-[3]. The wavelets possess multidimensional characters and are able to adjust their scale to the nature of the signal features. Singularities and irregular structures in signal waveform often carry important information from an informatics-theoretic point of view. The WT analysis provides a kind of mathematical "microscope" to zoom in or zoom out on those interesting structures [3]. Furthermore, wavelets can be orthonormal and are able to capture

This work was supported by National Science Council, Taiwan, R.O.C., under Grant NSC 91-2133-E-002-113.

J.-A. Jiang is with the Department of Bio-Industrial Mechatronics Engineering, National Taiwan University, Taipei 106, Taiwan, R.O.C. (Telephone: +886-2-3366-5341, Fax:+886-2-2362-7620, e-mail: jajiang@ ccms.ntu.edu.tw).

P.-L. Fan and J.-Y. Sheu are with the Department of Electrical Engineering, Kuang-Wu Institute of Technology, Taipei, Taiwan:

C.-S. Chen is with the Department of Electrical Engineering, National Taiwan University, Taipei, Taiwan, R.O.C

C.-S. Yu is with Department of Electrical Engineering, Chung Cheng Institute of Technology, National Defense University, Taoyuan, Taiwan, R.O.C. deterministic features. Therefore, WT can decompose a signal into localized contributions labeled by so-called dilation and translation parameters. These parameters represent the information of different frequency component contained in the analyzed signals [3].

To monitor the quality of an electric power system, a sinusoidal waveform at a rated voltage magnitude and frequency is a proper index. Thus, any electric power disturbance (or fault) can be thought of as a deviation from that sinusoidal waveform. The other problem that should be noticed when a fault occurs on a power system is that fault current is almost greater than the pre-fault load current in any system element. A very simple and effective fault detecting principle is that of using the current magnitude as an indicator of a fault of the power system.

Since the wavelet transform with its ability allowing the localization both in time and frequency domain, some publications [4]-[6] introduced the wavelet transform as a tool to analyze power system disturbances. In this work, the authors apply the dyadic wavelet transform with Haar wavelets to analyze three phase-currents of a power system. By the multi-resolution analysis characteristics of the dyadic wavelet transform, the abrupt direct offset (DC) component of the current in a power system can be found and used to be a fault detection indicator. Meanwhile, the faulted-phase can also be identified. The proposed algorithm is implemented by using moving data window technique. The simulation studies show that this fault detection indicator is with fast response time, which provides an alternative approach for transmission line protection.

The organization of the paper is described as follow. First, the dyadic wavelet transform is introduced. Basic idea and the proposed algorithm are described in Section III. Then, the simulation studies are shown in Section IV. Finally, conclusions are given.

\section{DYADIC WAVELET TRANSFORM}

For measured data, the wavelet dilation and translation parameters are discretized dyadically as $a=2^{j}, b=2^{j} k$, where $j$ and $k$ are integers and the indices for scaling and translation, respectively. Thus, the $j$ th scale dyadic wavelet transform of $f(t)$ can be represented as [7] 


$$
\begin{aligned}
W f(j, k) & =\left\{f(t), \psi_{j, k}(t)\right\} \\
& =2^{-j / 2} \int_{-\infty}^{\infty} f(t) \psi\left(2^{-j} t-k\right) d t
\end{aligned}
$$

Here, the translation parameter $k$ determines the location of the wavelet in the time domain, whereas the dilation parameter $j$ determines the location in the frequency domain as well as the scale or extent of the time-frequency localization.

Any signal may be decomposed into its contributions in different regions of the time-frequency space by projecting it on the corresponding wavelet basis function. Fast algorithms for computing the wavelet decomposition are based on representing the projection of the signal on the corresponding basis function as a filtering operation. Thus, the coefficients at different scales may be obtained as

$$
\begin{aligned}
& A_{2 j}\{f(n)\}=\sum_{k=-\infty}^{\infty} h(k) A_{2 j-1}\{f(n-k)\} \\
& D_{2 j}\{f(n)\}=\sum_{k=-\infty}^{\infty} g(k) A_{2 j-1}\{f(n-k)\}
\end{aligned}
$$

Explicitly, $A^{j}$ and $D^{j}$ are obtained from $A^{j-1}$ by

$$
\begin{aligned}
& A^{j}=\sum_{k} h_{k-2 L} A^{j-1} \\
& D^{j}=\sum_{k} g_{k-2 L} A^{j-1}
\end{aligned}
$$

where $A^{j}$ and $D^{j}$ are $j$ th discrete approximation and discrete details, and $h(k)$ and $g(k)$ are discrete low-pass filter and high-pass filter, respectively corresponding to $h(t)$ and $g(t)$. The original data is considered to be the scaling function coefficients at the finest scale, i.e. $A^{0}$. Thus, the decomposition can be schematically described as fig.1:

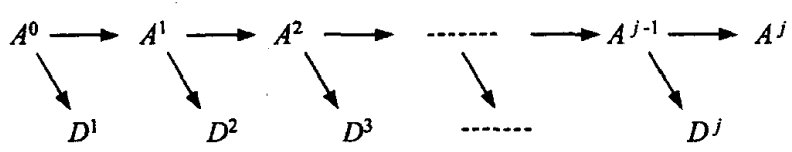

Fig. 1 Decomposition structure for Haar wavelet transformation.

To reconstruct the signal from its wavelet decomposition is just to run recursion algorithm in the reverse conjugates of filter $h(k)$ and $g(k)$. The reconstruction algorithm is therefore, also a pyramid algorithm, using the same filter coefficients as the decomposition. Signal reconstruction can be presented as

$$
A^{j-1}=\sum_{k} h_{k-2 L} A^{j}+\sum_{k} g_{k-2 L} D^{j}
$$

and it can be represented schematically by fig. 2 .

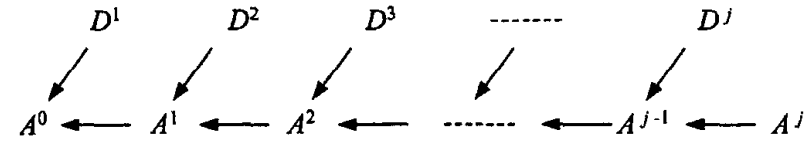

Fig. 2 Reconstruction structure for Haar wavelet transformation.

Therefore, when the spectrum is described by wavelet coefficients (discrete approximations and discrete details), it is easy to distinguish the noise component from the component containing relevant information to reconstruct the initial spectrum. An increasing value of dilation parameter $j$ corresponds to a greater degree of smoothing of the original function. Thus, the de-noised data can be obtained by simply choosing one of the discrete approximations. In addition, of all discrete detail there must be a discrete detail whose frequency is higher than original data and lower than the high frequency noise. Hence, this detail could represent the resolved signals and the number of the components in the original data can be obtained from this detail.

\section{BASIC IDEAS AND ALGORITHM DESIGN}

When a fault occurs in transmission lines, the abrupt DC component of the current in a power system can be used to be a fault detection indicator. First, the authors performed one experiment with the signal waveform described by equation (7).

$$
x(t)=\sin (\omega t)+d c
$$

where $d c$ is the added DC offset.

A Haar wavelet is used as the analyzing wavelet filter in this work. The Haar mother function can be expressed by equation (8)

$$
\operatorname{haar}(t)=\left\{\begin{array}{cc}
1, & 0 \leq t<\frac{1}{2} \\
-1, & \frac{1}{2}<t \leq 1 \\
0, & \text { otherwise }
\end{array}\right.
$$

In this work, the number of points for an observation window is 32 . Since the number of discrete data is 32 , the level of the decomposition is $5\left(2^{5}=32\right)$. The Haar wavelet transform of the signal $x(t)$ is shown in fig. 3. Apparently, the coefficient $a_{5}$ represents the DC offset of the input signal. Thus, the coefficient $a_{5}$ is a proper fault indicator of the postfault current of a power system when the fault occurrences. When the sampling rate is 16 points per cycle, the level of the decomposition is $4\left(2^{4}=16\right)$ and so on. Thus, using the Haar wavelet transform to extract to the $\mathrm{DC}$ component, and then the fault can be detected. 

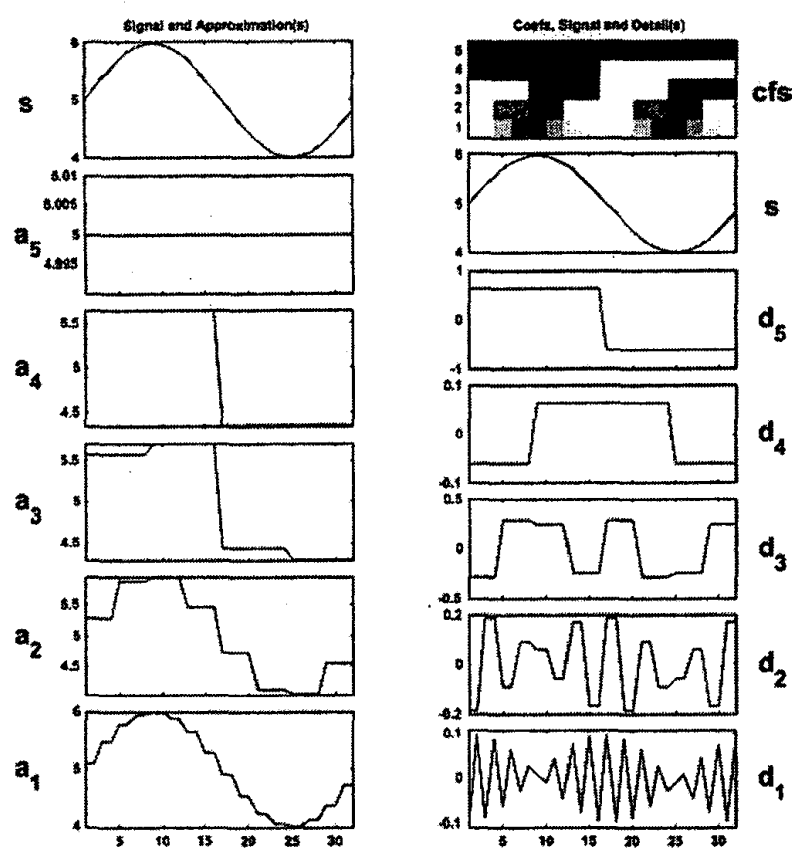

Fig. 3 Discrete approximations and discrete details of $\operatorname{signal} \sin (\omega t)$ with $d c=$ 5 (decomposition level $=5$ ).

For practical usage, the absolute value of the DC component, denoted by $\left|a_{5}\right|$, is used as a fault detection indicator. According to the above descriptions, the proposed fault detection and faulted-phase selection algorithms are described below. For every moving data window, the DC components of three phase-currents are extracted using the Haar wavelet, respectively. Thus, we can obtain three fault detection signals $\left|a_{5}\right|_{m}$, where $m$ represents ' $a$ ', 'b', 'c' phase respectively. Under steady state conditions, these DC components are zero. When a fault occurs, large transient DC components are generated. Thus, incorporating with a threshold setting (TH), the fault can be detected. Moreover, monitoring the three fault detection signals respectively, the faulted-phase is identified. For example, when a phase-' $a$ ' to ground fault occurs, the magnitude of fault detection signal of phase-' $a$ ', $\left|a_{5}\right|_{a}$, will be greater than the pre-defined threshold.

However, the signals of phase-' $b$ ' and ' $c$ ' are lower than the threshold. The overall flowchart of the proposed algorithm is shown in fig. 4.

\section{SIMULATION EXPERIMENTS}

\section{A. Simulation System}

For evaluating the performance of the proposed algorithm, the authors adopt MATLAB/Simulink for fault data generation and algorithm implementation. Fig. 5 depicts the

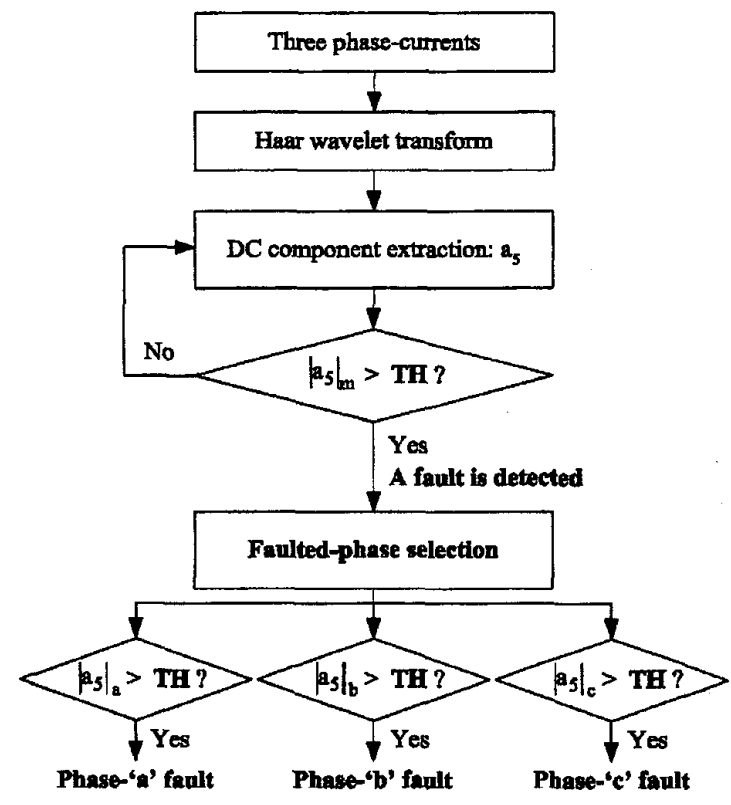

Fig. 4 The overall flowchart of the proposed algorithm.

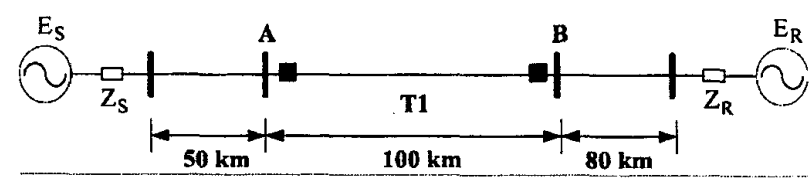

Fig. 5 The single-line diagram of the simulated system.

single-line diagram of the simulated system, which is a 345 $\mathrm{kV}$ transposed transmission line system. Distributed line model is utilized in this simulation. T1 is a protected line segment. The parameters of the adopted line and two Thévenin's equivalent source circuits are same as in [8]. In this work, the phase difference between $E_{S}$ and $E_{R}$ is set as 20 degrees. A second-order low-pass Butterworth pre-filter whose cutoff frequency is $360 \mathrm{~Hz}$ first filters the faulted current waveforms. The three-phase current signals at relay location, which is at bus ' $A$ ', are sampled at $1920 \mathrm{~Hz}$ (i.e. 32 points per-cycle). The selected simulation results are briefly summarized below.

\section{B. Example Studies}

In this part, the authors select three cases to illustrate the performance of the proposed fault indicator under internal fault events.

1) Case 1

First, a phase-' $a$ ' to ground fault is selected as a simulation case whose fault path resistance is 1 , fault location is at 0.5 (p.u.), and fault inception angle is zero degree refers to ' $a$ 'phase voltage wave, respectively.

Fig. 6 depicts the behaviors of fault detection indicator $a_{5}$ 


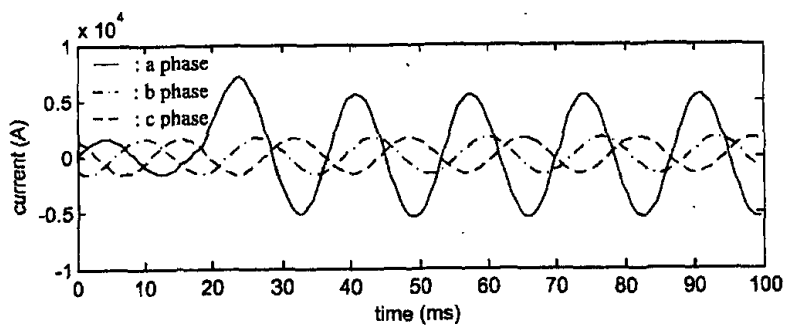

(a)

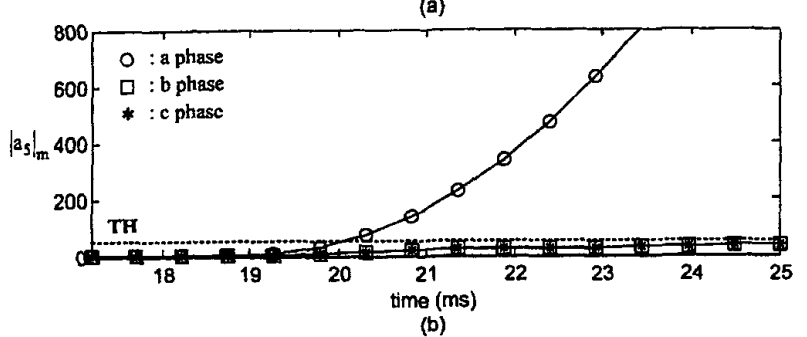

Fig.6 (a) Simulated three-phase current waveforms and (b) fault detection indicator $\left|a_{5}\right|_{m}$ for an internal fault case 1 .

for the internal fault case. Fig. 6-(a) shows the simulated current waveforms of phases $a, b$, and $c$. It is obvious that the current of phase-a contains large decaying dc offset component. In this paper, the threshold setting of TH is chosen to be 50. Fig. 6-(b) indicates the fault detection indicator $a_{5}$ of a-phase increases as the fault occurs while those of b-phase and c-phase still remain zero approximately. That $\left|a_{5}\right|$ value will be greater than threshold setting after fault occurrence such that the fault can be detected. It is also easy to distinguish fault occurrence in which phase from fault detection indicator $\mathbf{a}_{5}$.

2) Case 2

Fig. 7 depicts the behaviors of fault detection indicator $a_{5}$ for a phase-'a' to ground fault whose fault inception angle is changed to be 90 degrees. At this time, the current contains no decaying dc offset component. Since the transient current signal still contains DC component after fault occurrence, the proposed fault indicator $\left|a_{s}\right|$ can detect the internal fault. As shown in this figure, the faulted-phase ' $a$ ' is correctly identified.

3) Case 3

This subsection presents the responses of the proposed scheme with respect to an ' $a$ '-' $b$ ' phase to ground fault. The fault position is set at $40 \mathrm{~km}$ away from bus $A$. The fault resistance is 5 and fault inception angle is zero degree.

Fig. 8 describes the response curves of the fault detection indicator $a_{5}$ under this double-phase fault event. As seen from fig. 8-(b), it is worthy noted that the DC component of the fault current $\left(a_{5}\right)$ increases in a-phase but that decreases in $b$ phase. Fig. 8-(c) shows that the proposed fault indicator fast detects the fault and correctly identifies the faulted-phase.

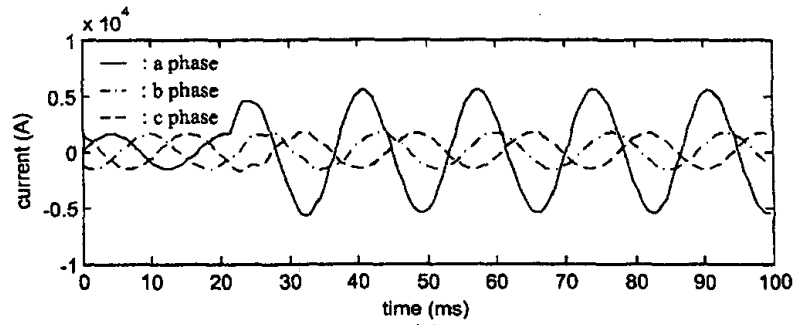

(a)

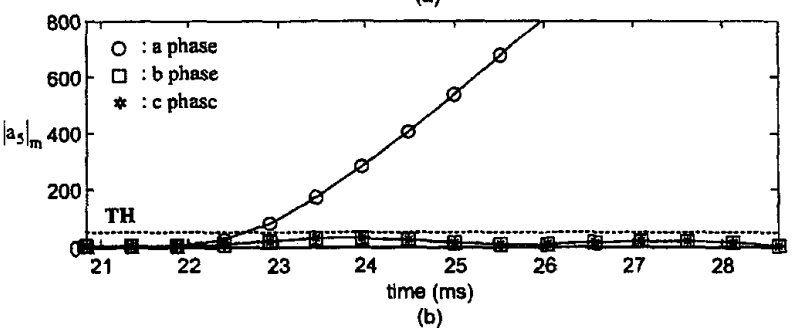

Fig.7 (a) Simulated three-phase current waveforms and (b) fault detection indicator $\left|a_{5}\right|_{m}$ for an internal fault case 2 .

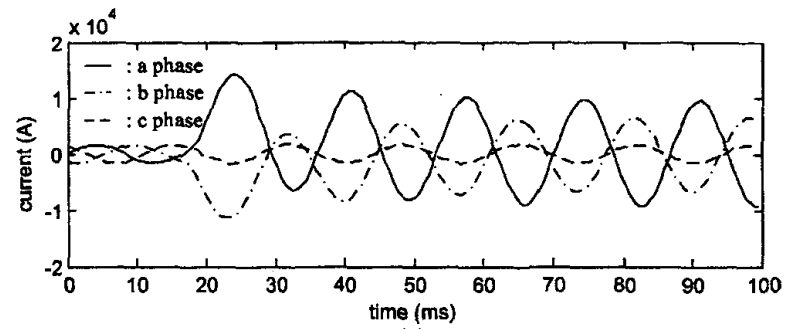

(a)

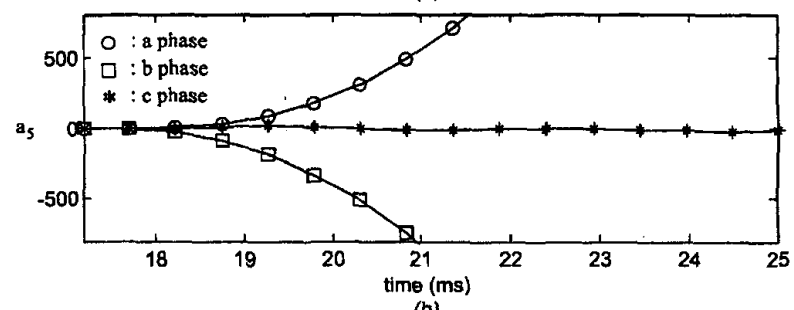

(b)

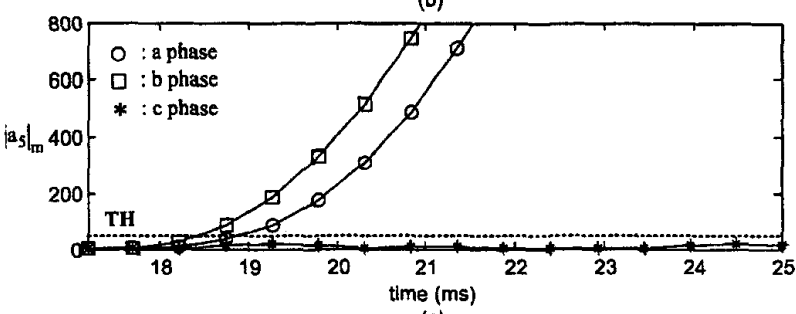

Fig.8 (a) Simulated three-phase current waveforms, (b) fault detection indicator $a_{5}$, and (c) fault detection indicator $\left|a_{5}\right|_{m}$ for an internal fault case 3.

The fault detection indicator $a_{5}$, which copes with the judgment of the fault occurrence, such an arrangement has 
verified that the proposed fault detection scheme can perform well in this study.

\section{Conclusions}

A dyadic wavelet transform based approach for transmission line protection is presented in this work. A simple and effective fault detecting approach based on dyadic wavelet transform is completed. A fault detection indicator based on the discrete approximation of the Haar wavelet is derived. The abrupt change of current component of the fault current for a power system can be detected by the dyadic wavelet transform by using the one-end current information as an input. The fault detection indicator also can achieve the task of faulted-phase selection. The simulation studies demonstrate that the proposed algorithm is feasible for transmission line protection.

\section{REFERENCES}

[1] O. Chaari, M. Meunier, and F. Brouaye, "Wavelet: a New Tool for the Resonant Grounded Power Distribution Systems Relaying", IEEE Trans. on Power Delivery, vol. 11, no.3, pp. 1301-1308, July 1997.

[2] R. K. Martinet, J. Morlet, and A. Grossmann, "Analysis of sound patterns through wavelet transforms", Int. J. Patt. Rec. Art. Intell. 1, 273-302, 1987.

[3] C. K. Chui, " Wavelet: A tutorial in theory and application," Academic Press, 1991.

[4] G. T. Heydt, and A. W. Galli, "Power quality problem analyzed using wavelets," IEEE Trans. on Power Systems, vol. 12, No 2, pp.869-915, Apr. 1997.

[5] T. B. Littler, and D. J. Morrow, "Wavelets for the analysis of power system disturbances," IEEE Trans. on Power Delivery, vol. 14, No 4, pp. 358-364, Apr. 1999.

[6] S. Huang, C. Hsieh, and C. Lien Huang," Application of Morlet wavelets to supervise power system disturbances," IEEE Trans. on Power Delivery, vol. 14, No. 1, pp. 235-243, January 1999.

[7] C. S. Burrus, R. A. Gopinath, and H.T. Guo, Introduction to Wavelets and Wavelet transform, Prentic Hall Press, 1998.

[8] J. A. Jiang, Y. H. Lin, J. Z. Yang, T. M. Too, C. W. Liu, “An Adaptive PMU Based Fault Detection/Location Technique for Transmission Lines, Part II: PMU Implementation and Performance Evaluation", IEEE Trans. on Power Delivery, vol. 15, No. 4, pp. 1136-1146, October 2000.

\section{BIOGRAPHIES}

Joe-Air Jiang (M'01) was born in Tainan, Taiwan, R.O.C., in 1963. He received the M.S. and $\mathrm{Ph} . \mathrm{D}$. degrees in electrical engineering from National Taiwan University, Taipei, Taiwan, R.O.C., in 1990 and 1999, respectively.

Currently, he is Assistant Professor of bio-industrial mechatronics engineering at National Taiwan University. From 1990 to 2001, he was with Kuang-Wu Institute of Technology, Taipei, Taiwan, R.O.C. His areas of interest are in computer relaying, mechatronics, and bio-effects of electromagnetic wave.

Ping-Lin Fan was born in Taipei, Taiwan, on December 29, 1961. He received the B.S. degree in electrical engineering and the M.S. degree in optical science from the National Central University, Chung-Li, Taiwan, in 1985 and 1987, respectively. He received Ph.D. degree in optical science from the same university in 2002. He is now an associate professor in electrical engineering of Kuang-Wu Institute of Technology. His research interests cover the design and application of mechatronics.

Ching-Shan Chen was born in Taichung, Taiwan, in 1976. He received the B.S. degree in electrical engineering from National Taiwan University of Technology and Science in 1998, and the M.S. and Ph.D. degrees in electrical engineering from National Taiwan University, Taipei, Taiwan, in 2000 and 2003 . Hs research interests include computer relaying and the application of phasor measurement units to power system protection.

Chi-Shan Yu was born in Taipei, Taiwan in 1966. He received his B.S. and M.S. degrees in electrical engineering from National Tsing Hua University in 1988 and 1990, and Ph.D. degree in electrical engineering from National Taiwan University in 2001. From 1991 to 2001, he has been with Kuang-Wu Institute of Technology, since 2002, he came to National Defense University, Chung-Cheng Institute of Technology, where he is assistant professor of electrical engineering. His research areas are in computer relaying and power system transient stability controller design.

Jin-Yi Sheu was born in Taipei, Taiwan, on April 19, 1956. He received the B.S. degree in physics from the Soochow University in 1978 and the M.S. degree in physics from the National Taiwan Normal University in 1980 , respectively. He received Ph.D. degree in optical science from the National Central University in 2002. He is now an associate professor in electrical engineering of Kuang-Wu Institute of Technology. His research interests cover the optical design and the image processing. 